\title{
Magnetization profiles at the upper critical dimension as solutions of the integer Yamabe problem
}

\author{
Alessandro Galvani, ${ }^{1}$ Giacomo Gori, ${ }^{2,3}$ and Andrea Trombettoni ${ }^{4,1,3}$ \\ ${ }^{1}$ SISSA and INFN, Sezione di Trieste, Via Bonomea 265, I-34136 Trieste, Italy \\ ${ }^{2}$ Institut für Theoretische Physik, Universität Heidelberg, D-69120 Heidelberg, Germany \\ ${ }^{3}$ CNR-IOM DEMOCRITOS Simulation Center and SISSA, Via Bonomea 265, I-34136 Trieste, Italy \\ ${ }^{4}$ Department of Physics, University of Trieste, Strada Costiera 11, I-34151 Trieste, Italy
}

\begin{abstract}
We study the connection between the magnetization profiles of models described by a scalar field with marginal interaction term in a bounded domain and the solutions of the so-called Yamabe problem in the same domain, which amounts to finding a metric having constant curvature. Taking the slab as a reference domain, we first study the magnetization profiles at the upper critical dimensions $d=3,4,6$ for different (scale invariant) boundary conditions. By studying the saddle-point equations for the magnetization, we find general formulas in terms of Weierstrass elliptic functions, extending exact results known in literature and finding new ones for the case of percolation. The zeros and poles of the Weierstrass elliptic solutions can be put in direct connection with the boundary conditions. We then show that, for any dimension $d$, the magnetization profiles are solution of the corresponding integer Yamabe equation at the same $d$ and with the same boundary conditions. The magnetization profiles in the specific case of the 4-dimensional Ising model with fixed boundary conditions are compared with Monte Carlo simulations, finding good agreement. These results explicitly confirm at the upper critical dimension recent results presented in [1].
\end{abstract}

\section{INTRODUCTION}

Within the field of critical phenomena, a research line that attracted considerable attention along the years is the study of critical systems in presence of boundaries [2, 3]. Among the motivations for such a study, the first is to correctly compare theoretical predictions with experimental results or numerical simulations coming from finite systems. In general, the introduction of a boundary alters thermodynamic properties and sub-leading corrections to bulk values have been extensively studied [4]. Techniques such as finite-size scaling can be used to extract critical exponents and other important quantities 5. In two-dimensional systems, the coefficient of the next-to-next-to-leading order in the free energy is related to the central charge of the theory [6] and the study of boundary conformal field theory developed in the last decades as a very active field of research [7].

A remarkable property emerging in critical bounded domains is that a given bulk universality class corresponds to several surface universality classes, making boundary critical phenomena a rich and well studied playground. In many cases, boundaries are the source of very interesting effects and phenomena. A major example is the thermodynamic Casimir effect: it has been studied thoroughly to obtain universal scaling function and Casimir amplitudes [8. The interest in the Casimir effect has shifted the attention from semi-infinite systems [9 12 to the geometry of a slab [13 17. Obtaining the critical magnetization profile [18, 19] is a crucial piece of information in this line of research.

The study of critical phenomena with a field theoretical approach 20 naturally highlights the role of the dimensionality which rules the relevance of fluctuations at the critical point. For $d$ matching the so-called upper critical dimension $d_{c}$ [5, 21] the critical theory becomes typically tractable, yielding in a controlled way the mean-field approximation and the typical logarithmic corrections on top of it $[22,23$. If critical exponents at the upper critical dimension are found to be just the mean-field ones, in bounded systems there is the possibility to study inhomo- geneous, space-dependent quantities and corrections due to the presence of boundaries.

Here we are going to focus on magnetization profiles of models at their upper critical dimension. They can be a useful starting point for calculation of critical magnetization profiles at lower dimensions, as they give the background around which (non-logarithmic) fluctuations can be added. Moreover, an advantage of being at the upper critical dimension is that one can obtain, by a saddlepoint treatment, analytical expressions to be compared with numerical results.

Our goal in this paper is threefold. First, we obtain magnetization profiles in a unified framework for different boundary conditions, by considering the analytic structure of the saddle-point solutions. This will allow us to recast known results in a more accessible and compact way while deriving novel predictions, e.g., for percolation at its upper critical dimension. For the Ising model in four dimensions we test the findings obtained by the discussed approach by comparing them with results of Monte Carlo simulations.

Another main objective is to provide a geometric interpretation of the results for the critical magnetization profiles at the upper critical dimension. The main idea is to write the field theory equations for the magnetization profiles as a variational solution of a geometric problem. In this way, our aim of finding the critical profiles is explicitly put in connection with the solution of a celebrated problem in differential geometry: the Yamabe problem 24, which amounts to finding a metric, in the same conformal class of another given metric, that makes the scalar curvature constant. The Yamabe problem in its various generalizations has been the object of intense mathematical work in the last decades [25, 26. It is also related to general relativity, when one looks for solutions of Einstein field equations which are conformally flat or more generally within a conformal class 27. A discussion of the connection between the (mean-field) LandauGinzburg equations and the Hilbert-Einstein action functional for pure gravity in presence of cosmological term is presented in 28 . 
As a third motivation, the present study intends to explicitly verify the validity at the upper critical dimension of results presented in 11. There, a hypothesisreferred to as "uniformization" - is put forward to relate the critical magnetization profile of a bounded domain with a metric factor $\gamma(\mathbf{x})$, which is the solution of a fractional Yamabe equation in the same domain. This metric is used to compute distances between points, which are then used to construct spin-spin correlation functions. In this "critical geometry" approach, the points close to the boundary are put at larger distances by inflating locally and isotropically the original metric. In [1] the uniformization hypothesis was supported by numerical simulations of the three-dimensional Ising model, given the lack of analytical results for this model. It is important to notice that below the upper critical dimension a nonvanishing anomalous dimensions $\eta$ arises: in order to take this into account, the Yamabe equation has to be modified, by introducing a fractional power of the Laplacian operator, obtaining the fractional Yamabe equation [29]. But, at the upper critical dimension $\eta=0$ and the fractional Yamabe equation reduces to the standard Yamabe equation, which for clarity we call the integer Yamabe equation, since in it only the usual Laplacian enters. Therefore the study of critical magnetization profiles at $d=d_{c}$ allows us to test, analytically and numerically, the validity of the "critical geometry" approach in a solvable case.

We observe that the relation between critical magnetization profiles and the integer Yamabe equation holds in any bounded domain. We specialize to the slab geometry since it is convenient for both analytical calculations and simulations and for its relevance for the Casimir effect. We will comment on the general case when useful for our presentation.

The plan of the paper is the following: first we will consider Landau-Ginzburg actions with marginal interaction, for various dimensions: in each case, we will obtain the saddle-point equation at the corresponding upper critical dimension; solutions will be given for the possible boundary conditions (BC). These equations will then be generalized through the introduction of the Yamabe equation. After solving it for a slab in arbitrary dimension, we recover the previous solutions. The theoretical magnetization profiles are then compared to the numerical solution of the Ising mean field equation, and to the results of a Monte Carlo simulation of the four-dimensional Ising model.

\section{MAGNETIZATION PROFILES}

In the following, we study exact solution for the critical magnetization profiles for models living at their upper critical dimension. We write the action of a scalar field with marginal coupling and $\mathbb{Z}_{2}$ symmetry as

$$
S=\int d^{d} x\left[\frac{1}{2} \phi(-\triangle) \phi+\frac{1}{2} \mu^{2} \phi^{2}+g c_{d}\left(\phi^{2}\right)^{\frac{d}{d-2}}\right],
$$

where the field $\phi=\phi(\mathbf{x})$ with $\mathbf{x} \in \Omega$ and $\Omega \subset \mathbb{R}^{d}$ is a bounded domain. As usual, the operator $-\triangle$ is the positive definite Laplacian. Notice that the exponent of the potential term is integer for $d=3,4$, which are the dimensions we are going to focus on. The case of percolation, considered later, has to be treated separately with the introduction of a similar action in $d=6$. The action can be generalized to a vector field $\vec{\phi}$ with $N$ components: in this case, $\phi^{2}$ in the potential term is replaced by $\sum_{i=1}^{N} \phi_{i}^{2}$. The factor in the interaction constant

$$
c_{d} \equiv \frac{(d-2)^{2}}{8}
$$

has been chosen for convenience, as it will appear later. Since in $d$ dimensions the field has scaling dimension $\Delta_{\phi}=\frac{d-2}{2}$, the interaction term in (1) is written so that the action is at the upper critical dimension. The mass term $\propto \phi^{2}$ vanishing at the critical point is also included [5, 21].

The saddle-point equation for the action (1) at the critical point reads

$$
\triangle m(\mathbf{x})=g \frac{d(d-2)}{4} m(\mathbf{x})^{\frac{d+2}{d-2}}
$$

with $m(\mathbf{x})=\langle\phi(\mathbf{x})\rangle$.

We will now specialize the study of the saddle-point approximation for the action (1) for the physical dimensions taking the values $d=3,4$, and 6 . This is firstly due to their physical relevance, but also for the striking mathematical properties they display only for these values of $d$. The peculiarity amounts to the fact that they are the only dimensions where the magnetization profiles can be expressed as suitable powers of the Weierstrass elliptic function $\wp$ (see Appendix A and [30] for reference). Before moving to the different cases $d=3,4$, and 6 in Secs. IIB IID we discuss the different boundary conditions we are going to treat.

\section{A. Boundary conditions}

Let us start from the Ising model. We have different choices of boundary conditions on a slab; forcing the spins on the two boundaries to be aligned (fixed boundary conditions, FBCs) corresponds to diverging order parameter at the boundaries. One could have ++ or -- FBCs (due to symmetry, we consider only the ++ case). If the spins on the two boundaries are antiparallel, then one has +FBCs.

The corresponding, conformally invariant, boundary condition in the field theory is $\langle\phi\rangle= \pm \infty$. Of course, on a lattice the order parameter cannot diverge: the magnetization at the boundaries will be \pm 1 . For the ++ FBCs, the value at the center of the slab, however, will scale with the system size as $L^{-\Delta_{\phi}}$, so one can rescale the magnetization in a way that it is constant at the center. In the thermodynamic limit, it then diverges at the slab edges. It is this rescaled form which is accessible via field theory.

Another way to understand it is the following: if the lattice magnetization profile were prolonged a few sites beyond the boundary, these singularities would appear. This concept is quantified by the extrapolation length $a_{L}$ 2]: if the boundaries where we fixed the spins are at $x=0$ and $x=L$, after we fit the resulting profile $m(x)$, we would see divergences at $m\left(-a_{L}\right)$ and at $m\left(L+a_{L}\right)$. In 
the $L \rightarrow \infty$ limit, $a_{L} \rightarrow 0$ and the magnetization diverges at the boundaries.

One can also fix the spins on one boundary to the value +1 and the spins on the other boundary to the value -1 (+- FBCs). The corresponding magnetization profile vanishes in the center, so either half of this profile could be obtained by fixing the spins on one boundary, and leaving the other boundary free (mixed boundary conditions, +0 .)

We clarify that in the following, in agreement with action (1), we do not consider an external magnetic field, except for the one needed to fix the values of the spins at the boundaries. Therefore open boundary conditions (OBCs) are trivial, in the sense that the magnetization at the critical point has to vanish. In Appendix $\mathrm{C}$ we discuss the case of OBCs with a suitably chosen scaling magnetic field, providing an illustration of the usefulness of the calculation developed in this section and a further interesting perspective on the mathematical structure of the solutions.

From the scaling dimension $\Delta_{\phi}$ of the order parameter, we can also obtain the behavior near the boundary (see Table I)

\begin{tabular}{c|c|c|c}
\hline BC & Lattice & $m(x \rightarrow 0)$ & $m(x \rightarrow L)$ \\
\hline++ & $\uparrow \ldots \uparrow$ & $x^{-\Delta_{\phi}}$ & $(L-x)^{-\Delta_{\phi}}$ \\
+- & $\uparrow \ldots \downarrow$ & $x^{-\Delta_{\phi}}$ & $-(L-x)^{-\Delta_{\phi}}$ \\
+0 & $\uparrow \ldots 0$ & $x^{-\Delta_{\phi}}$ & $L-x$ \\
\hline
\end{tabular}

Table I: Labels for the possible boundary conditions, with the corresponding spin configuration and behavior of the continuous profile near the boundaries.

If we were to consider $O(N)$ models with $N>1$, there would be an additional degree of freedom in the choice of boundary conditions: the angle between the spins on the two boundaries. The magnetization becomes then a vector in the plane spanned by the boundary spins, and the saddle-point becomes a system of two equations. So, while the +- solution is specific to the Ising model, the ++ one is valid for any $O(N)$ model with parallel boundary spins. Here we focus on conformally invariant boundary conditions, which are homogeneous on the slab plates. Other boundary conditions are possible, such as the one considered in 31 to enforce a topological excitation.

\section{B. $\phi^{4}$ theory in four dimensions}

In four dimensions the action (1) reads

$$
S=\int d^{4} x\left[\frac{1}{2} \phi(-\triangle) \phi+\frac{1}{2} \mu^{2} \phi^{2}+\frac{1}{2} g \phi^{4}\right] .
$$

A corresponding model in the lattice is of course the Ising model. Since the theory is at the upper critical dimension, we proceed by writing the saddle-point equation. The saddle-point equation for the action at the critical point $\mu=0$ is

$$
\triangle m(\mathbf{x})=2 g m(\mathbf{x})^{3}
$$

We are interested in solving Eq. (5) in the case of a slab domain $[0, L] \times \mathbb{R}^{3}$. The magnetization $m(\mathbf{x})$ depends only on the transverse direction $x$, so the Laplacian $\triangle$ reduces to $\partial_{x}^{2}$. The different solutions for various boundary conditions will be analyzed and compared in the rest of the this section. In Sec. VI we will compare the analytical results so obtained with Monte Carlo simulations for the Ising model on a four-dimensional slab geometry with fixed boundary conditions.

Notice that given a solution $m(x)$ of (5) we can generate other solutions by translation and rescaling ruled by the parameters $x_{0}, \beta$, and $\lambda$, all of them being possibly complex:

$$
m(x) \rightarrow \beta m\left(\lambda\left(x-x_{0}\right)\right), \quad g \rightarrow g \beta^{-\frac{4}{d-2}} \lambda^{2} .
$$

This allows us to change the domain or coupling constant as desired; for convenience we set $g=1$. In general such scaling will alter the boundary conditions, however scaling invariant conditions, i.e., $m=0, \infty$, are left unchanged. We remark that the scaling (6) also applies to other values of $d$ and it will thus be useful in the next subsections.

The general solution to Eq. (5) (with $g=1$ ) is

$$
m(x)=\lambda \wp_{l}\left(\lambda\left(x-x_{0}\right)\right)^{1 / 2},
$$

where $\wp_{l}$ is the Weierstrass $\wp$ function with invariants $\left(g_{2}, g_{3}\right)=(1,0)$. This is the so-called lemniscatic case. When it is written in terms of the complex variable $z$ as $\wp_{l}(z)$, it corresponds in the complex plane to a square lattice, with a real half-period $\omega_{l}=\Gamma\left(\frac{1}{4}\right)^{2} /(4 \sqrt{\pi})$.

The Weierstrass elliptic $\wp$, whose basic properties are refreshed in Appendix A, is a doubly periodic function. Within one of its domains in the complex plane, it has a double pole and two zeros possibly coinciding (as here): taking segments with poles and/or zeros as their endpoints gives solutions with various boundary conditions, as can be seen in Fig. 1. Along the segments the function has to be real or have a constant phase (which can removed) in order to be interpreted as magnetization profiles.

The corresponding (unscaled) solutions are

$$
\begin{array}{lr}
m_{++}(x)=\wp_{l}(x)^{1 / 2}, & x \in\left(0,2 \omega_{l}\right), \\
m_{+0}(x)=\wp_{l}\left(x e^{i \pi / 4}\right)^{1 / 2} e^{i \pi / 4}, & x \in\left(0, \sqrt{2} \omega_{l}\right] .
\end{array}
$$

By extending the $m_{+0}(x)$ solution to the interval $x \in$ $\left(0,2 \sqrt{2} \omega_{L}\right)$ we indeed generate a solution $m_{+-}$connecting the + and - boundary states. This reflects the $\mathbb{Z}_{2}$ symmetry of the model as it is apparent in the saddlepoint equation (5) with $m(x) \rightarrow-m(x)$.

\section{C. $\phi^{6}$ theory in three dimensions}

A $\phi^{6}$ theory has upper critical dimension $d_{c}=3$. The steps of the previous section can be repeated in this case. We start from the Landau-Ginzburg action at the critical point, where the couplings of the $\phi^{2}$ and $\phi^{4}$ terms vanish:

$$
S=\int d^{3} x\left[-\frac{1}{2} \phi(-\triangle) \phi+\frac{g}{8} \phi^{6}\right] .
$$



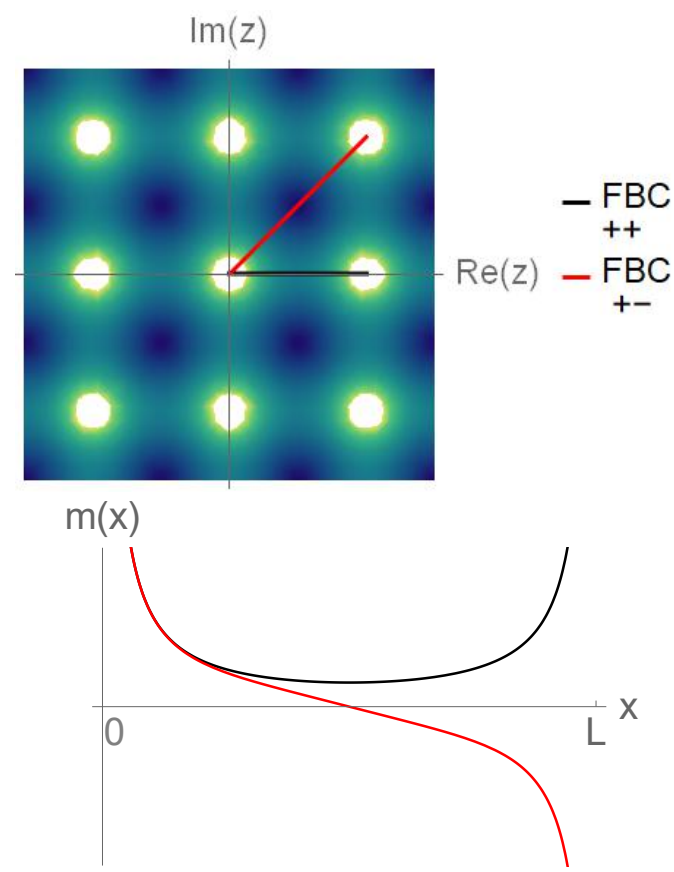

Figure 1: $d=4$ case. Above: modulus of the square root of the lemniscatic elliptic function, poles are white and zeros blue. Below: magnetization profiles for the different boundary conditions discussed in the text.

On the slab geometry $[0, L] \times \mathbb{R}^{2}$, we get the saddle-point equation

$$
m^{\prime \prime}(x)=\frac{3}{4} g m^{5}
$$

where again due to the scaling property (6) we set $g=1$. The solution is

$$
m(x)=\lambda^{1 / 2} \wp_{e}\left(i \lambda\left(x-x_{0}\right)\right)^{-1 / 2} .
$$

In the present case, the invariants of the Weierstrass function $\wp_{e}$ are $\left(g_{2}, g_{3}\right)=(0,1)$, from which its real halfperiod is $\omega_{e}=\Gamma\left(\frac{1}{3}\right)^{3} /(4 \pi)$.

The above case $\wp_{e}$ of the Weierstrass function is the socalled equiharmonic case, where the lattice used to define the elliptic function is the regular triangular tiling of the plane; see Fig. 2 .

Remarkably, the solution for $d=6$, presented in the next subsection, will turn out to be dual to this one. Again, looking at the poles and zeros of $m(z)$, we obtain solutions for the possible boundary conditions:

$$
\begin{aligned}
& m_{++}(x)=\wp_{e}\left(\omega_{e}+i x\right)^{-1 / 2}, \quad x \in\left(-\frac{\omega_{e}}{\sqrt{3}}, \frac{\omega_{e}}{\sqrt{3}}\right), \\
& m_{+0}(x)=\wp_{e}\left(x e^{i \pi / 6}\right)^{-1 / 2} e^{-i \pi / 6},
\end{aligned}
$$

These solutions could also be expressed through Jacobi elliptic functions, as was done for the ++ solution in [32], through Fisher-De Gennes theory [33]. Again due to $\mathbb{Z}_{2}$ invariance, reflected in the $m(x) \rightarrow-m(x)$ symmetry in the saddle-point equation, the $m_{+0}(x)$ can be extended further in the interval $x \in\left(-\frac{2}{\sqrt{3}} \omega_{e}, \frac{2}{\sqrt{3}} \omega_{e}\right)$ yielding a $m_{+-}(x)$ profile.

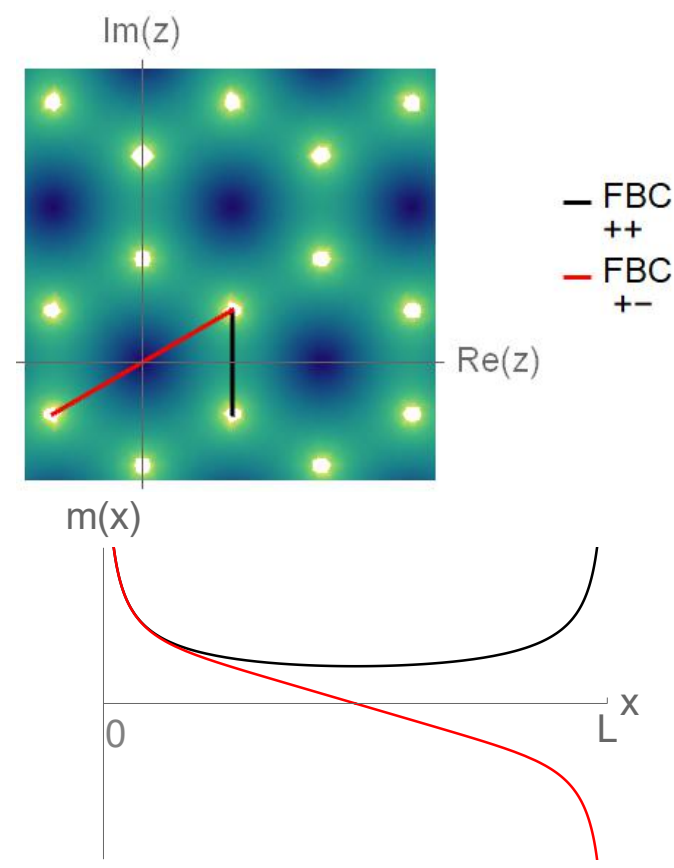

Figure 2: As in Fig. 1, but here for $d=6$, the modulus of $m(z)$ in the complex plane, with the solutions for the different boundary conditions.

\section{D. $\phi^{3}$ theory in six dimensions}

In $d=6$, we consider an action with cubic potential:

$$
S=\int d^{6} x\left[\frac{1}{2} \phi(-\triangle) \phi+2 g \phi^{3}\right],
$$

which is used to describe percolation at the upper critical dimension [34, 35]. This action is not obtained directly by plugging $d=6$ into (1), so it differs from the previously considered $d=3,4$ cases; since the potential is odd, it lacks $\mathbb{Z}_{2}$ symmetry. This implies the absence of opposing (+-) boundary conditions, which becomes clear when one thinks of the possible boundary conditions for percolation: one can either force a boundary to belong to the percolating cluster $(+)$, or leave it free, but opposing boundaries no longer make sense. However, we can still find a solution connecting the + and 0 boundary states. The saddle-point equation now is

$$
m^{\prime \prime}(x)=6 g m(x)^{2} .
$$

The general solution is (taking $g=1$ as in the previous cases) the function $\wp_{e}$ :

$$
m(x)=\lambda^{2} \wp_{e}\left(\lambda\left(x-x_{0}\right)\right),
$$

meaning that the profiles are just the square of the reciprocal of the $\phi^{6}$ results, as evidenced in Fig. 3. Now the double pole in the origin directly yields the expected divergence for the $m_{++}$order parameter profile, since the dimension of the field is $\frac{d-2}{2}=2$. The profiles are:

$$
\begin{array}{ll}
m_{++}(x)=\wp_{e}(x), & x \in\left(0,2 \omega_{e}\right), \\
m_{+0}(x)=\wp_{e}\left(x e^{i \pi / 6}\right) e^{i \pi / 3}, & x \in\left(0, \frac{2 \omega_{e}}{\sqrt{3}}\right] .
\end{array}
$$




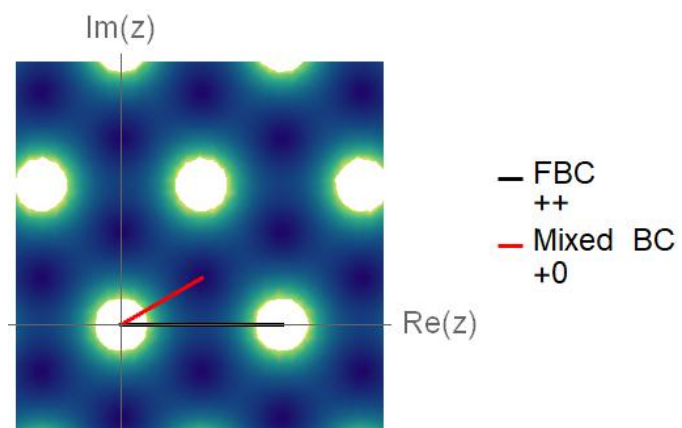

$m(x)$

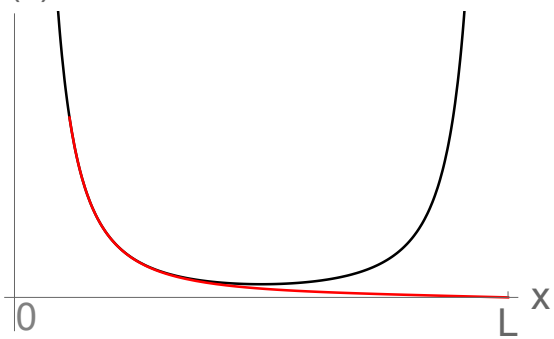

Figure 3: The zero and pole structure for $d=6$ is inverted with respected to Fig. 22. What would be the +- profile now ends at 0 , giving the mixed boundary condition +0 .

\section{THE YAMABE EQUATION}

In the following we provide the solution to the saddlepoint equation for $\phi^{2 m}$ theories, valid for critical dimensions $d_{c}=\frac{2 m}{m-1}$ (in this general case, they will not possess the analytic properties displayed by the $d=3,4,6$ cases discussed above).

In order to introduce a geometric interpretation of the critical magnetization profile, we notice that it is possible to re-write the saddle-point equation in a convenient form. Since the scaling dimension of the order parameter field is $\Delta_{\phi}=\frac{d-2}{2}$, we introduce a function $\gamma(\mathbf{x})$ which acts as a point-dependent scale. We then write

$$
m(\mathbf{x})=\alpha \gamma(\mathbf{x})^{-\Delta_{\phi}}=\alpha \gamma(\mathbf{x})^{-\frac{d-2}{2}},
$$

where $\alpha$ is a constant, to be fixed.

The main point of this section, as well as the main result of the paper, is that the solution $m(\mathbf{x})$ of the saddlepoint equation (3), once rescaled as in (17), can be written as

$$
(-\triangle) \gamma(\mathbf{x})^{-\frac{d-2}{2}}=-\frac{d(d-2)}{4} \gamma(\mathbf{x})^{-\frac{d+2}{2}},
$$

where $\alpha$ in 17 is chosen to be $\alpha=g^{(2-d) / 4}$ so that the right-hand side has the correct coefficient to obtain what is known as the Yamabe equation. Equation (18) can be also recast in the form

$$
1-|\vec{\nabla} \gamma(\mathbf{x})|^{2}+\frac{2}{d} \gamma(\mathbf{x}) \triangle \gamma(\mathbf{x})=0 .
$$

The Yamabe equation has the geometric interpretation of a constraint on the curvature of a manifold. This equation can be viewed as a special case of the more general fractional Yamabe equation, which contains a fractional Laplacian, allowing it to describe theories with nonvanishing anomalous dimension as done in [1. In the following, whenever we mention the Yamabe equation, we are referring to the integer one, with standard Laplacian, as first introduced in [24] .

To understand how a geometric problem emerges from one-point functions of a bounded system at criticality, we discuss the connection of the obtained results with the "uniformization" hypothesis put forward in [1].

\section{A. Uniformization hypothesis and the Yamabe equation}

The main property a system typically gains at its critical points is conformal invariance [36, 37]. Heuristically, this means that every point and every region of the system look the same. Introducing a boundary clearly breaks this property. The question addressed in [1] is then: is there a way to recover it? If a metric is introduced which sets the boundary at an infinite distance, then we would no longer have a distinction between points close to the boundary and points deep in the bulk. The only changes of the euclidean metric that we can allow are conformal transformations, since the system must still be locally euclidean. This means that the choice of metric reduces to the choice of a function $\gamma(\mathbf{x})$ which sets a local scale:

$$
\delta_{i j} \rightarrow g_{i j}=\frac{\delta_{i j}}{\gamma(\mathbf{x})^{2}}
$$

$\delta_{i j}$ being the flat metric, i.e., the identity matrix, and $i, j=1, \ldots, d$.

Constraints on the function $\gamma$ have to be imposed. Since we have a curved space, we should look into the various quantities that describe its curvature, the most obvious one being the Ricci scalar curvature. The main hypothesis in [1] is that the metric must make a bounded critical system as uniform as possible: this means making the scalar curvature constant. This curvature would have to be negative, since spaces with positive curvature, like spheres, lack boundaries. The simplest examples of space with constant negative curvature are the Poincaré half plane and disk models.

Starting from the metric with an unknown $\gamma(x)$, one can compute the Christoffel symbols, from which one gets the Ricci tensor, and finally the Ricci scalar:

$$
R=\sum_{i, j=1}^{d} R_{i j} g^{i j}=\kappa, \quad \kappa<0 .
$$

Without losing generality, we can set $\kappa=-1$. We can now write this condition as an equation for the factor $\gamma(\mathbf{x})$, getting 18$)$, supplemented by the condition $\gamma(\mathbf{x})=0$ at the boundaries of the domain $\Omega$. This is enough to get solutions which, close to the boundary, are proportional to the euclidean distance from it. The distance from any point to the boundary, computed with this metric, is therefore infinite, as desired.

To frame it more generally, the Yamabe problem consists in finding a metric in the conformal class of another given metric for a smooth Riemann space, that makes the scalar curvature constant [25]. A solution has been proven to exist for any such space, provided it is compact. 
The two-dimensional limit of 18 gives the Liouville equation

$$
(-\triangle) \log \gamma(\mathbf{x})=-\gamma(\mathbf{x})^{-2} .
$$

The Yamabe equation is a nonlinear differential equation. There are a few cases where the Yamabe equation has simple solutions: e.g., for a ball of radius $R$ in any dimension, one finds $\gamma=\frac{R^{2}-|\mathbf{x}|^{2}}{2 R}[1,26]$; the case of the slab geometry will be treated in the next section.

One-point functions and the scale factor transform similarly under a dilation of the system $\Omega \rightarrow \lambda \Omega$ :

$$
\left\langle\phi_{\lambda \Omega}(\lambda \mathbf{x})\right\rangle=\lambda^{-\Delta_{\phi}}\left\langle\phi_{\Omega}(\mathbf{x})\right\rangle, \quad \gamma_{\lambda \Omega}(\lambda \mathbf{x})=\lambda \gamma_{\Omega}(\mathbf{x}),
$$

where $\Delta_{\phi}$ is the scaling dimension of the field. Therefore, once $\gamma(\mathbf{x})$ is known, all one-point functions are determined up to a constant $\mathrm{C}$ :

$$
\langle\phi(\mathbf{x})\rangle=\frac{\mathcal{C}}{\gamma(\mathbf{x})^{\Delta_{\phi}}} .
$$

As an example, for a half space in any dimension, with $x_{1}>0$ and $\left\{x_{2}, \ldots, x_{d}\right\} \in \mathbb{R}^{d-1}$, we have $\gamma(\mathbf{x})=x_{1}$, so

$$
\langle\phi(\mathbf{x})\rangle=\frac{\mathcal{C}}{x_{1}^{\Delta_{\phi}}},
$$

reproducing a standard result of boundary conformal field theory [7.

The introduction of $\gamma(\mathbf{x})$ in (17) should now be clearer: the mean-field equation for a (multi-)critical of the $O(N)$ model is equivalent to the Yamabe equation at the corresponding upper critical dimension. Once the scale factor $\gamma(\mathbf{x})$ is obtained by solving the Yamabe equation, the magnetization is recovered through (24).

\section{ANALYTICAL RESULTS FOR THE SLAB GEOMETRY}

For the slab geometry we can actually obtain the solution for general dimension $d$ in implicit form. We denote the solution of the Yamabe equation (18) with $\gamma_{d}(x)$ to emphasize the dependence on the dimension $d$. For convenience we assume $x \in[0,2]$ (i.e., $L=2$ ), so that the center of the slab is in $x=1$.

For $x \in[0,1]$ and $++\mathrm{FBC}$, the relation between $x$ and $\gamma_{d}$ is given in terms of the ${ }_{2} F_{1}$ hypergeometric function. From the Yamabe equation one straightforwardly finds

$$
x\left(\gamma_{d}\right)={ }_{2} F_{1}\left(\frac{1}{d}, \frac{1}{2} ; 1+\frac{1}{d} ;\left(\frac{\gamma_{d}}{\gamma_{m}}\right)^{d}\right) \gamma_{d} .
$$

By reflecting $x$ around 1: $x \rightarrow 2-x$ the other branch is obtained. The constant

$$
\gamma_{m}=\frac{\Gamma\left(\frac{1}{2}+\frac{1}{d}\right)}{\sqrt{\pi} \Gamma\left(1+\frac{1}{d}\right)}
$$

is the (maximum) value acquired by the conformal factor at the center of the slab. Equation (26) is valid for any $d \geq 2$, including the cases $d=4,6,3,2$ and also real values of $d$. Notice that a slab configuration cannot be defined for $d<2$. The formulas for other boundary conditions are written below.

We pause here to comment that one sees a (minor) advantage of using the Yamabe equation in the form 19. instead of using directly the saddle-point equation (3). The latter, when written in the slab geometry, gives rise to the so called Emden-Fowler equation 38. The latter, for a function $m(x)$, reads in its canonical form $m^{\prime \prime}(x)=A x^{\mathcal{N}} m(x)^{\mathcal{M}}[38$, with our case corresponding evidently to $\mathcal{N}=0$ and $\mathcal{M}=(d+2) /(d-2)$. The case $\mathcal{N}=0$ can be solved by quadrature [38, writing the solution in term of an integral and for certain values of $\mathcal{M}$ the corresponding analytical expressions are tabulated [38. However, solving the Yamabe equation (19) and using the procedure prescribed in ordinary differential equations textbooks (or directly Mathematica) one finds that the solution is given by the inverse of the hypergeometric function ${ }_{2} F_{1}$ for any $d$. Therefore, rewriting $m$ in terms of $\gamma$ via the equation (17) may also help to find easier analytical solutions, as the case of the slab geometry shows. In Appendix C, we discuss how to relate the result 26, to different boundary conditions, including open boundary conditions with an external magnetic field.

We can see what the general result (26) simplifies to when we substitute $d=4,3,6,2$ :

$d=4$ : the inverse of 26 is $\wp_{l}(x)^{-1 / 2}$, the lemniscatic Weierstrass function used in (5), see Appendix B.

$d=6$ : the inverse is the equiharmonic elliptic function $\wp_{e}(x)$ as seen in 15 .

$d=3$ : this case is dual to the $d=6$ case, since the solution here is simply the square root of the reciprocal of the previous solution, after an appropriate translation; this is clarified in Appendix $\mathrm{C}$

$d=2$ : the value $d=2$ cannot be directly chosen in the Yamabe equation (18): a limit has to be performed, yielding the Liouville equation 22 . One may then assume that plugging $d=2$ into 26) will give an incorrect result. Surprisingly, that is not the case: for $d=2$, the function ${ }_{2} F_{1}\left(\frac{1}{2}, \frac{1}{2} ; \frac{3}{2} ; \gamma^{2} \frac{\pi^{2}}{4}\right) \gamma$ reduces to the inverse sine: indeed, $\gamma_{2}(x)=\frac{2}{\pi} \sin (\pi x / 2)$.

This agrees with and generalizes previous results: profiles for ++ and +- boundary conditions were found for $d=4$ in [18, and for $d=2$ in [39]; the ++ profiles for $d=2,3,4$ are also found in 32 . In both 18 and [32] Jacobi functions were used. Switching to Weierstrass functions allowed us in the present paper to write profiles for various dimensions in a compact way, retrieving the previously listed known results, finding new results for all the conformally invariant boundary conditions. We also obtain new results for all the critical magnetization profiles in $d=6$.

\section{SOLUTION OF LATTICE MEAN-FIELD EQUATIONS}

In this section we discuss the solution of the mean-field equations for the Ising model in a slab geometry directly on the lattice. The goal is to see how lattice profiles can be accurately described by a continuous function from 


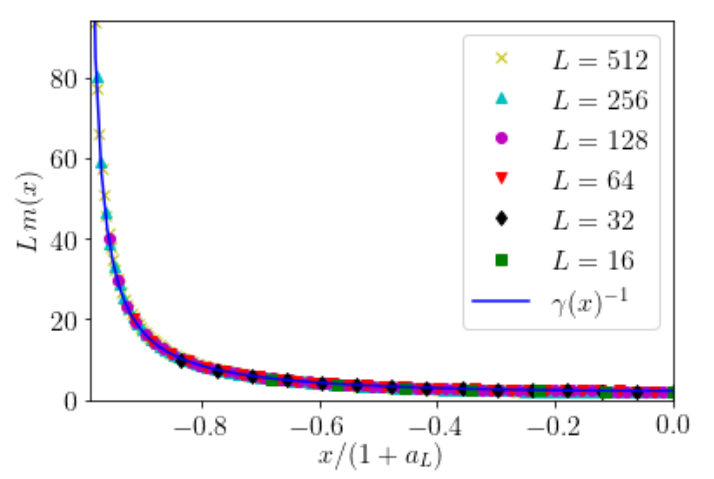

Figure 4: Collapse of the mean-field magnetization profiles. Since they are symmetric around the center of the slab, we plot only the left half. Each set of magnetization values has been rescaled by a factor $L$, while the $x$ coordinate has been rescaled through its extrapolation length.

field theory in the thermodynamic limit. For this reason in this section we take the slab coordinate $x$ to be an integer variable, going from 1 to $L$, and then take $L \rightarrow$ $\infty$. In this calculation it is not necessary to specify the number of sites in the directions perpendicular to $x$, since we can assume that the probability distribution function $P$ of any spin depends only on its transverse coordinate $x$. For this reason we put

$$
P\left(s_{i}\right)=\frac{1+m_{x}}{2} \delta_{s_{i}, 1}+\frac{1-m_{x}}{2} \delta_{s_{i},-1},
$$

where $s_{i}= \pm 1$ is the Ising variable in the site $i$ having $x$ as coordinate along the direction of the slab.

We can find the discrete magnetization profile $m_{x}=$ $\left\langle s_{i}\right\rangle$ minimizing the free energy. The mean-field equation $22]$ is

$$
m_{x}=\tanh \left(\beta \sum_{j}\left\langle s_{j}\right\rangle\right)
$$

This is the same equation used to find the inverse critical temperature $\beta_{c}$ in the bulk, i.e. in the case of no boundaries. Having set the coupling $J$ to 1 for convenience, it is $\beta_{c}=1 / 2 d$. Of course, it differs from the critical temperature of the actual Ising lattice, see the next Section.

In a four dimensional slab with $d=4$, this becomes

$$
m_{x}=\tanh \left[\beta\left(6 m_{x}+m_{x+1}+m_{x-1}\right)\right] .
$$

For the case of FBC $m_{1}=m_{L}=1$, this equation can be solved iteratively until the difference between the two sides is smaller than a fixed threshold.

The value of the magnetization at the center of the slab decays, as the system size increases, as $1 / L$. Since profiles for different sizes must have the same functional shape at the critical point, we rescale them by multiplying each profile by its corresponding size $L$. This also clarifies the correspondence between fixing the boundary spins to +1 in the lattice model and diverging boundary conditions in the field theory. As the size increases, the rescaled boundary magnetization grows proportional to $L$.

At the same time, as $L$ increases, the extrapolation length $a_{L}$ [2] decreases, meaning that the point where the profile diverges gets closer to the lattice boundaries. In the limit $L \rightarrow \infty, a_{L} \rightarrow 0$ and the rescaled $m(0)=$ $m(L) \rightarrow \infty$.

Once the rescaling is done, we clearly see a collapse of the various profiles, showing that we are at the critical point. The corresponding results are in Fig. 4. For large $L$, the extrapolation length vanishes and the meanfield profile coincides with the saddle-point solution. We also studied the solutions of the lattice mean-field equations for +0 boundary conditions, finding a similarly good agreement between them and the solution of the corresponding Yamabe equation.

\section{MONTE CARLO SIMULATION OF THE $4 d$ ISING MODEL ON A SLAB}

The previous predictions have been obtained by performing the saddle-point approximation on the action (1). It is very well known that in four dimensions for the $\phi^{4}$ theory, and in general at the upper critical dimension, the critical exponents are the mean-field ones [5, 21, 23]. This is routinely exploited in conformal bootstrap calculations for the bulk geometry, where in $d=4$ the critical theory is Gaussian [0]. However, in a bounded domain - to the best of our knowledge - there is no proof that the critical magnetization profile in the thermodynamic limit given by the saddle-point approximation is exact in $d=4$ for the $\phi^{4}$ theory, although it is expected. For this reason we decided to numerically test the saddle-point findings by Monte Carlo simulations and validate our predictions via numerical experiments. In order to obtain a numerical check of the magnetization profiles, we will concentrate on the Ising model on the slab geometry with FBC. Notice that explicit numerical investigations for high dimensions of phenomena arising from inhomogeneities are rather sparse especially if compared to two-dimensional systems. Three dimensional models did receive of course attention, and basic predictions from scale and conformal invariance have recently been tested [41, 42.

We performed Monte Carlo simulations of the Ising model at its upper critical dimension, 4 , in a slab of sizes $L$ in the transverse direction, and $4 L$ in the other three directions; $L$ ranges from 16 to 56 . The FBC are implemented by fixing the spins to the same $(+1)$ or opposite $( \pm 1)$ on the two faces in the transverse direction, while the other directions have periodic boundary conditions. We can then expect the ++ and +- magnetization profiles from (8). The critical inverse temperature $\beta_{c}$ is taken to be $\beta_{c}=0.1496927$ from [43, see also [44, 45].

The simulation uses the standard Metropolis algorithm, whose moves are single spin flips. After a thermalization time, we measured the average magnetization and local energy along hyperplanes parallel to the boundaries. As explained in Secs. II and $/ \mathrm{V}$, since the spins are fixed at the edge faces and one is at the bulk critical temperature, the magnetization profile will start from the value 1 at one boundary, will decrease as one approaches the center, should reach a value of order of $1 / L$, and then rise again up to 1 in the other slab boundary. One can rescale the numerical data in a way that the value of the magnetization at the center is constant, meaning that the boundary magnetization increases with $L$. 


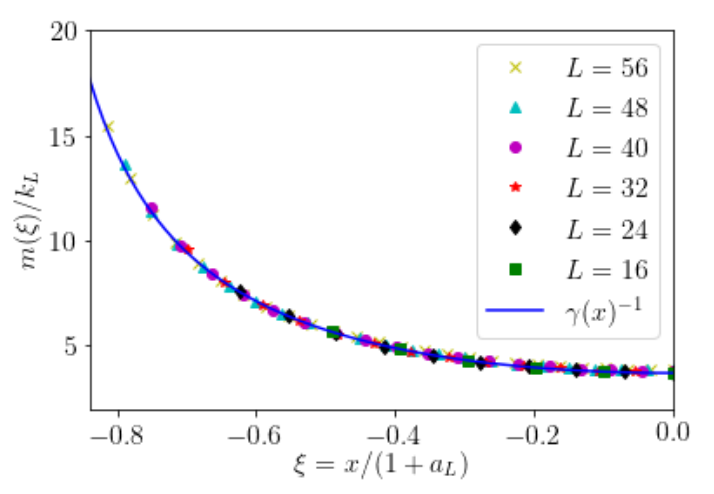

Figure 5: Collapse of the critical magnetization profiles in the four-dimensional Ising model for slabs of different sizes with ++ boundary conditions. Again, we plot only half the profile. Each set of points has been rescaled with a multiplicative constant and its extrapolation length, obtained from the fit 31

\begin{tabular}{cc}
\hline$L$ & $a_{L}$ \\
\hline 16 & 0.2465 \\
24 & 0.1724 \\
32 & 0.1314 \\
40 & 0.1070 \\
48 & 0.0884 \\
56 & 0.0404 \\
\hline
\end{tabular}

Table II: Decreasing extrapolation lengths as the size increases $(++\mathrm{BC})$.

The magnetization data are then fitted with

$$
m(x)=k_{L}\left[\gamma\left(\frac{x}{1+a_{L}}\right)\right]^{-1}
$$

where $k_{L}$ is a multiplicative constant roughly proportional to the system size, and $a_{L}$ is the extrapolation length, with the index ${ }_{L}$ denoting the dependence on the size. The extrapolation length shrinks the lattice profile so that the divergence of the order parameter would appear a few sites beyond the edges [2, 5]. We see that it becomes smaller as the size grows, meaning that larger sizes describe a larger portion of the saddle-point profile. In Fig. 5 and 6, we plot the magnetization profiles obtained for different sizes, compared with the prediction from (24). The magnetization $m(x)$ for different sizes are plotted as functions of the respective rescaled variable $\xi=x /\left(1+a_{L}\right)$, which highlights the collapse. Simulations with +0 boundary conditions would look like half of the profile shown in Fig. 6.

Despite qualitative agreement between the prediction and the numerical data, the distance between raw data points and the theoretical curve is larger than the estimated numerical error (in Figs. 5 and 6 smaller than the point sizes). This is to be expected: while (24) gives the correct mean-field behavior, observables at the upper critical dimension also include some logarithmic corrections. To account for them, we proceed as in 43. At the critical temperature, the main finite size corrections

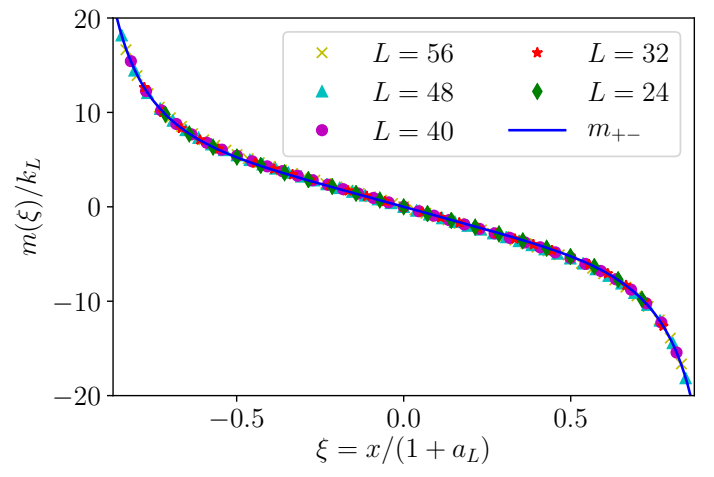

Figure 6: Collapse of the critical magnetization profiles in the four-dimensional Ising model for +- boundary conditions.

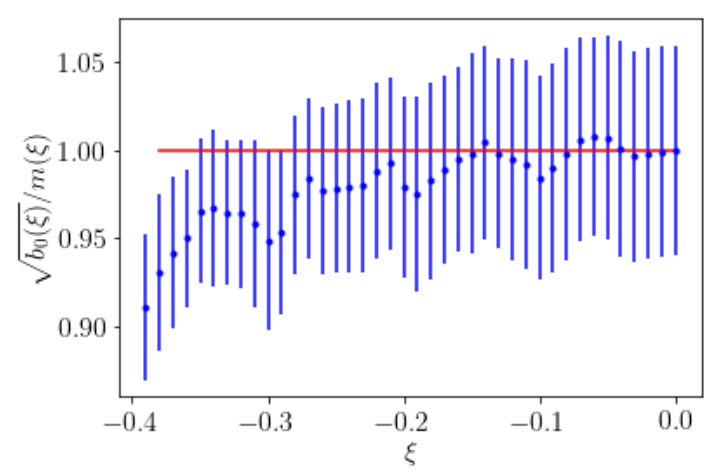

Figure 7: Ratio of the square root of the fit parameter $b_{0}$ and the expected magnetization $(++\mathrm{BC})$. The boundary $x=-1$ correspond to $\xi=-1 /\left(1+a_{L}\right)$, and the first few points close to the boundary must be discarded, so the plot starts from $\xi=-0.39$.

to the bulk magnetization take the form

$$
m=c \frac{(\ln L)^{1 / 4}}{L} \sqrt{b_{0}+\frac{b_{1}}{\ln L}+\frac{b_{2}}{(\ln L)^{2}}},
$$

where terms with higher powers of $1 / \ln L$ have been neglected. In our case, the magnetization is a function of $x$ (or rather of the rescaled coordinate $\xi$ ), and therefore so are $b_{0}, b_{1}$ and $b_{2}$. For every $\xi$ we find the values of $b_{0}(\xi)$, $b_{1}(\xi)$ and $b_{2}(\xi)$ which best fit $m(\xi, L)$, seen as a function of $L=\{16,24,32,40,48,56\}$ 48.

In order to compare with the analytical prediction 8 for $++\mathrm{BC}$, the constant $c$ in $(32)$ is chosen so that $\sqrt{b_{0}(0)}=m(0)$. We then plot, in Fig. 7. the ratio $\sqrt{b_{0}(\xi)} / m(\xi)$, and we see that it remains close to 1 . As we approach the boundaries, the value of the first logarithmic correction $b_{1}$ grows, explaining the deviation of the ratio from 1 . Larger values of $b_{1}$ simply imply that it is numerically harder to measure the values predicted by (24) near the boundaries, as it requires simulating even larger systems.

From $\xi=-1$ to $\xi=-0.4$ we decided not to plot the ratio $\sqrt{b_{0}(\xi)} / m(\xi)$ since, due to the values of the extrapolation length, for these $\xi$ 's we do not have points for each of the considered values of $L$. We remind that we are in the left part of the slab geometry, $\xi=0$ corresponding to 
the center and $\xi=-1$ to the left edge (we discretize $\xi$ in steps of 0.01). We notice that by fitting the points from the magnetization using $(32)$ without the logarithmic corrections one obtains a clearly worse estimate of the $\chi^{2}$, confirming the validity of the fitting function 32 . Moreover, for any $\xi$ we find $b_{0}>b_{1} / \ln L_{\max }>b_{2} /\left(\ln L_{\max }\right)^{2}$, where $L_{\max }=56$ is the maximum value of $L$ we were able to simulate. This shows that each successive term is a smaller correction to the infinite-size term, proportional to $b_{0}$. The standard deviations $\sigma$ in Fig. 7 are obtained by fitting the magnetization points without weighting them with their respective errors. The point for $\xi=0$ has ratio $\sqrt{b_{0}(\xi=0)} / m(0)=1$ by construction, and with standard deviation - determined as explained above $-\sigma=0.04$. The final result, depicted in Fig. 7 . shows that $\sqrt{b_{0}(\xi)}$ is compatible with $m(\xi)$ within $\sigma$ for $-0.32 \leq \xi \leq 0$ and within $2 \sigma$ for $-0.39 \leq \xi \leq-0.33$. To obtain more data in the range $-1<\xi \leq-0.4$ one should have larger sizes. For the +- data reported in Fig. 6 similar results have been obtained.

The conclusion is that for the data we have, the analytical predictions is in agreement with the Monte Carlo numerical results within $2 \sigma$.

\section{CONCLUSIONS}

In this paper we studied the magnetization profiles of models with marginal interaction for different (scale invariant) boundary conditions. Taking the slab as a reference domain, we first studied the magnetization profiles at the upper critical dimensions $d=3,4,6$. We put the zeros and poles of the Weierstrass elliptic solutions (written as a function of a complex variable) in connection with the different boundary conditions. We found general formulas in terms of Weierstrass elliptic functions, extending known results and finding new ones for percolation.

We then studied the connection between the critical magnetization profiles in a general domain and the solutions of the Yamabe problem in the same domain and with the same boundary conditions.

The solutions of the Yamabe equation solve the socalled Yamabe problem, which amounts to finding a metric having constant curvature. This shows that the saddle-point equation for the critical magnetization at the upper critical dimension is equivalent to a purely geometric description, where the euclidean metric is conformally altered to obtain a space with uniform negative curvature. In the slab geometry, using the Yamabe equations we derived analytical expressions for critical magnetization profile as inverse hypergeometric functions valid for any dimension $d \geq 2$. The expressions are valid for fixed boundary conditions, but with suitable shifts in the argument of the slab coordinate variable we can obtain the corresponding solutions for the other considered boundary conditions. Lattice mean-field results for the slab geometry in $d=4$ have been also presented.

The magnetization profiles in the specific case of the four-dimensional Ising model with fixed boundary conditions in the slab are compared with Monte Carlo simulations, finding good agreement.

The presented results explicitly confirm at the upper critical dimension recent results presented in [1. There, general correlation functions of the magnetization field in a bounded domain have been conjectured to be related to the solution of a fractional Yamabe problem, which with vanishing anomalous dimension becomes the integer problem here considered.

As next step at the upper critical dimension, a worthwhile subject of investigation would be to study spinspin correlation functions in the four-dimensional case for bounded domains, and check if and how they depend on the geometric distance defined by the metric obtained from the solution of the integer Yamabe problem in the considered domain. Another important question is how the present approach can treat or be extended to other surface universality classes, such as the special transitions 2. We also observe that the present paper confirms that the Yamabe approach correctly describes fixed boundary conditions, while more work is needed to understand whether it can be extended to other boundary conditions such as open boundary conditions.

The well-known statistical models have a small anomalous dimension in three dimensions. Hence, one could be led to the study of a fractional Yamabe problem in which the exponent of the Laplacian is close to an integer value, to try and obtain a solution as a perturbation around the solution of the integer Yamabe equation. This would be interesting both to compare with other perturbative approaches and for the challenging task to develop a suitable perturbative schemes to solve the fractional Yamabe equation relevant for critical models below the upper critical dimension. Its effectiveness would then have to be compared with standard perturbative techniques.

We also wonder if the analytic structure found can be extended beyond the slab geometry at the upper critical dimension treated here. When the domain is not of the form $[0, L] \times \mathbb{R}^{d-1}$, is it still possible, given a magnetization profile, to obtain profiles for other boundary conditions by some process of continuation?

If we instead consider the fractional Yamabe problem (in the slab to begin with) can we use similar techniques to the ones described here to provide a solution? The solution to this problem was already found in [1] via generalization of AdS/CFT-borne scattering techniques [46] providing a beautiful mathematical framework. When searching for explicit solutions to the fractional Yamabe problem within this framework, however, one is faced with great mathematical challenges, as it requires to both solve the vacuum Einstein equations for a metric in $d+1$ dimensions, and then find the solution of a nonlinear eigenvalue problem in the obtained metric space. It would be very appealing to have simpler schemes available.

More concretely, the next step will be to study a three dimensional system, a case which is also numerically more accessible, to test the predictions of the fractional Yamabe equation against data below $d_{c}$. It is then worth asking whether the profiles obtained from $\gamma(\mathbf{x})$ and the unknown function that describes two-point correlations can be approximately recovered in a perturbative manner, as an expansion around the four dimensional solutions.

Acknowledgements: We thank J. Cardy, N. Defenu, S. Dietrich, T. Enss, A. Gambassi and A. Squarcini for useful discussions at various stages of this work. GG is sup- 
ported by the Deutsche Forschungsgemeinschaft (DFG, German Research Foundation) under Germany's Excellence Strategy EXC 2181/1 - 390900948 (the Heidelberg STRUCTURES Excellence Cluster).

\section{Appendix A: Reminders about Weierstrass elliptic functions}

Elliptic functions appear in numerous areas of physics. They get their name from their property of being the inverse of elliptic integrals. A complex function of one complex variable $f(z)$ is called elliptic if it is meromorphic (its only singularities are poles) and is doubly periodic,

$$
f\left(z+2 \omega_{1}\right)=f(z), \quad f\left(z+2 \omega_{2}\right)=f(z),
$$

with $\omega_{1} / \omega_{2} \notin \mathbb{R}$. $\omega_{1}$ and $\omega_{2}$ are called half-periods. The double periodicity induces a tessellation of the complex plane in parallelograms. It is therefore sufficient to know the values of the function within one of these parallelograms, say the one with vertices $0,2 \omega_{1}, 2 \omega_{2}$ and $2 \omega_{1}+2 \omega_{2}$.

The Weierstrass $\wp$ function is probably the most intuitive elliptic function to construct, starting from the definition and the requirement of having only a double pole within each cell:

$$
\begin{aligned}
\wp(z)=\frac{1}{z^{2}}+ & \sum_{\substack{m, n=-\infty \\
(m, n) \neq(0,0)}}^{\infty}\left(\frac{1}{\left(z+2 m \omega_{1}+2 n \omega_{2}\right)^{2}}-\right. \\
& \left.-\frac{1}{\left(2 m \omega_{1}+2 n \omega_{2}\right)^{2}}\right) .
\end{aligned}
$$

Instead of the half periods, the Weierstrass function can be identified with another pair of numbers, $g_{2}$ and $g_{3}$, called invariants. They are the lowest order coefficients in the Laurent expansion of $\wp$ around 0 :

$$
\wp(z)=\frac{1}{z^{2}}+\frac{g_{2}}{20} z^{2}+\frac{g_{3}}{28} z^{4}+O\left(z^{6}\right) .
$$

The invariants can be obtained from the half periods as

$$
\begin{gathered}
g_{2}=60 \sum_{(m, n) \neq(0,0)} \frac{1}{\left(2 m \omega_{1}+2 n \omega_{2}\right)^{4}} \\
g_{3}=140 \sum_{(m, n) \neq(0,0)} \frac{1}{\left(2 m \omega_{1}+2 n \omega_{2}\right)^{6}} .
\end{gathered}
$$

The importance of the invariants comes from the fact that they appear in the differential equation that the Weierstrass function obeys:

$$
\wp^{\prime 2}(z)=4 \wp^{3}(z)-g_{2} \wp(z)-g_{3}=0
$$

The particular cases encountered in the text are:
- $\left(g_{2}, g_{3}\right)=(1,0)$, called lemniscatic elliptic function $\wp_{l}$, which gives orthogonal semiperiods $\omega_{1}=\omega_{l}=$ $\Gamma\left(\frac{1}{4}\right)^{2} /(4 \sqrt{\pi}), \omega_{2}=i \omega_{1}$, that tessellate the complex plane with squares.

- $\left(g_{2}, g_{3}\right)=(0,1)$, the equiharmonic case $\wp_{e}$, with $\omega_{1}=\omega_{e}=\Gamma\left(\frac{1}{3}\right)^{3} /(4 \pi)$ and $\omega_{2}=\frac{1}{2}(\sqrt{3} i-1) \omega_{1}$, which produces a tessellation of parallelograms each made of two equilateral triangles.

\section{Appendix B: Inverse functions for the lemniscatic and equiharmonic Weierstrass elliptic functions}

In the main text explicit solutions for the nonlinear ODE $m^{\prime \prime}(x) \propto m^{\frac{d+2}{d-2}}$ have been derived for special values of $d$ in terms of the lemniscatic and equiharmonic Weierstrass elliptic functions $\wp_{l}$ and $\wp_{e}$. When solving the equation for generic $d$ a solution has been presented in implicit form. This allows us to write the following inversion formulas for $\wp_{l}$ and $\wp_{e}$ :

$$
\begin{aligned}
& \wp_{l}^{-1}(x)=\frac{1}{\sqrt{x}}{ }_{2} F_{1}\left(\frac{1}{4}, \frac{1}{2} ; \frac{5}{4} ; \frac{1}{4 x^{2}}\right) \\
& \wp_{e}^{-1}(x)=\frac{1}{\sqrt{x}}{ }_{2} F_{1}\left(\frac{1}{6}, \frac{1}{2} ; \frac{7}{6} ; \frac{1}{4 x^{3}}\right) .
\end{aligned}
$$

These two results can be obtained by considering different representation of the elliptic integrals $\int \mathrm{d} x \frac{1}{\sqrt{4 x^{3}-1}}$ and $\int \mathrm{d} x \frac{1}{\sqrt{4 x^{3}-x}}$.

\section{Appendix C: Open boundary conditions with a scaling magnetic field}

In the main text we did not consider the possibility of leaving the spins on both boundaries to take any value, that is open boundary conditions (OBC, which we will label 00). The reason is that in this case, the magnetization profile is trivially $m(x)=0$ throughout the slab since the system is at the critical temperature (see, e.g. [47]). In order to obtain nontrivial magnetization profiles for OBCs, one needs to introduce a magnetic field. The action at the critical point thus reads:

$$
S=\int d^{d} x\left[\frac{1}{2} \phi(-\triangle) \phi+g c_{d}\left(\phi^{2}\right)^{\frac{d}{d-2}}-h(x) \phi(x)\right]
$$

and the corresponding saddle-point equation is

$$
m^{\prime \prime}(x)=g \frac{d(d-2)}{4} m(x)^{\frac{d+2}{d-2}}-h(x)
$$

The scaling dimension of the magnetic field is given by $\Delta_{h}=d-\Delta_{\phi}$. In the language of the Yamabe approach, $\gamma(x)$ takes the role of the point-dependent length scale of the system, so a sensible choice would be to introduce a scaling magnetic field, given by an appropriate power of the conformal factor: $m(x) \propto \gamma(x)^{\Delta_{\phi}-d}$. One can then 

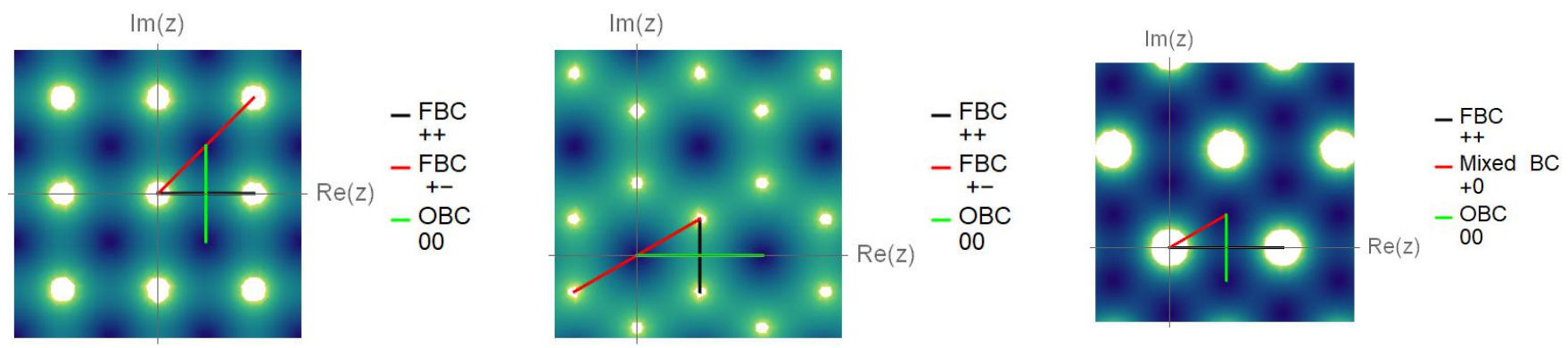

Figure 9: Pole and zero structure of the solutions of the saddle-point equations in $d=4,3,6$ respectively, with the addition of the 00 profile. The $d=3$ and $d=6$ structures are dual to each other: the 00 solution in $d=3$ corresponds to the ++ solution in $d=6$ and vice versa.

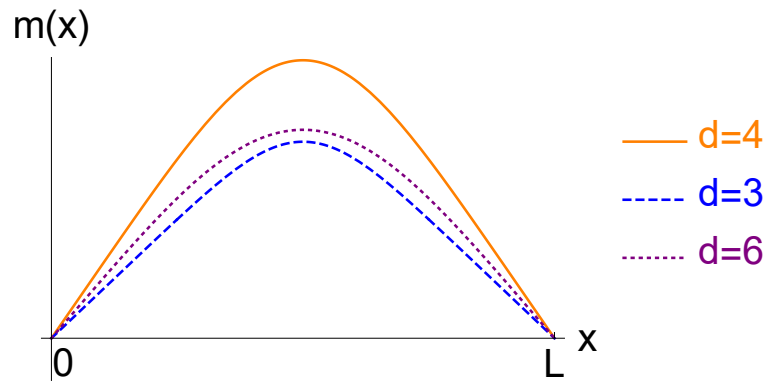

Figure 8: Magnetization profiles with $\mathrm{OBC}$ and a scaling magnetic field, in the three cases.

use (17) to replace $\gamma(x)$ with the magnetization profile itself. Remembering that $\Delta_{\phi}=\frac{d-2}{2}$, we get

$$
h(x) \propto m^{\frac{d+2}{d-2}} .
$$

In particular, choosing

$$
h(x)=g \frac{d(d-2)}{2} m^{\frac{d+2}{d-2}}
$$

Other magnetic fields could be chosen, however the corresponding magnetization profile would in general no longer be an elliptic function.

Let us focus on the $d=4$ solution. Numerical solutions on the Ising model with an external field confirmed the correctness of the solution $m_{00}$. The function $m_{00}$ is nothing but the reciprocal of the ++ solution (8), up to a constant: shifting the argument of the function by half a period has the same effect as taking its inverse, since both operations swap poles and zeros. This is because the fundamental domain of $\wp_{L}$ is a square, and the square lattice tessellation of the plane is self-dual, as seen in the left panel of Fig. 9. This peculiarity of the elliptic functions must reflect in a property of the saddle-point equation, valid only for $d=4$ and when the Laplacian reduces to an ordinary second derivative: if $\phi(x)^{-1}$ is a solution, then so is $\mathcal{K} \phi(x)$, for an appropriate $\mathcal{K}$. Starting from

$$
\frac{d^{2}}{d x^{2}} \phi(x)^{-1}=2 \phi(x)^{-3}
$$

we get

$$
\phi \phi^{\prime \prime}-2\left(\phi^{\prime}\right)^{2}+2=0
$$

we get that the solutions of $\sqrt{\mathrm{C} 2}$ are also solutions of the saddle-point equation without external field, but with $g$ in (3) replaces by $-g$. This means that these solutions also take the form of elliptic functions: in particular, they are obtained joining two zeros in the complex plane, as shown in Fig. 9 .

The magnetization profiles with the external field given by (C4) for $d=4,3,6$, plotted in Fig. 8 are the following:

$$
\begin{array}{lrl}
d=4: & m_{00}(x)=\wp_{l}\left(\omega_{l}+i x\right)^{1 / 2}, & x \in\left[-\omega_{l}, \omega_{l}\right] \\
d=3: & m_{00}(x)=\wp_{e}(x)^{-1 / 2}, & x \in\left[0,2 \omega_{e}\right] \\
d=6: & m_{00}(x)=\wp_{e}\left(\omega_{e}+i x\right), & x \in\left[-\frac{\omega_{e}}{\sqrt{3}}, \frac{\omega_{e}}{\sqrt{3}}\right]
\end{array}
$$

(where $\left.\phi^{\prime \prime}=\frac{d^{2}}{d x^{2}} \phi\right)$. Taking another derivative gives

$$
\frac{\phi^{\prime \prime \prime}}{\phi^{\prime \prime}}=3 \frac{\phi^{\prime}}{\phi} \text {, }
$$

and, after integrating and exponentiating, we are left with

$$
\phi^{\prime \prime}(x)=\mathcal{K} \phi(x)^{3},
$$

which is the saddle-point equation for $\sqrt{\frac{2}{\mathcal{K}}} \phi(x)$.

Similarly, the $d=3$ and $d=6$ solutions are dual to each other, as can be seen in Fig. 9. the poles in $d=6$ correspond to the zeros in $d=3$ and vice versa, linking the ++ solution in one case to the 00 solution in the other.

Finally, we can discuss how to relate the result 26 to different boundary conditions in any dimension. We write the magnetization via (17) as $m_{d}(x)=\gamma_{d}(x)^{-\Delta_{\phi}}=$ $\gamma_{d}(x)^{-\frac{d-2}{2}}$ where $\gamma_{d}(x)$ is given in 26$)$. If in $m_{d}(x)$ we set $d=3,4$, and 6 and aptly rescale the $x$ domain we recover the ++ boundary magnetization profiles given in Sec. III. This can be achieved by using the identities reported in Appendix B. The other profiles, referring to different boundary conditions, can be found by taking $x$ in 26 to be complex according to the following scheme: 


$$
\begin{array}{lr}
m_{++}(x)=m_{d}(x) & x \in(0,2), \\
m_{+0}(x)=m_{d}\left(x e^{i \pi / d}\right) e^{-i \pi \Delta_{\phi} / d} & x \in(0, \sec (\pi / d)], \\
m_{00}(x)=m_{d}(1+i x) & x \in[-\tan (\pi / d), \tan (\pi / d)],
\end{array}
$$

where, again, $m_{00}(x)$ solves the saddle-point equation with opposite sign. It is worth observing that the three BCs can be found by evaluating $\gamma$ on the right triangle $\mathbf{T}=(0,1,1+i \tan (\pi / d))$. Indeed the function $z(w)=$ $w^{1 / d}{ }_{2} F_{1}\left(\frac{1}{d}, \frac{1}{2} ; 1+\frac{1}{d} ; w\right)$ appearing in 26 is nothing but the Schwarz function mapping the upper-half-plane ( $w$ variable) onto $\mathbf{T}$ ( $z$ variable, called $x$ when real) keeping $z=0,1$ fixed. In the convenient variable $w$ we have that $\gamma=w^{1 / d}$ and $m=w^{-\Delta_{\phi} / d}=w^{-\frac{d-2}{2 d}}$. The above consideration should shed some light on the appearance of the Weierstrass functions $\wp_{e}, \wp_{l}$ in Sec. II and on the peculiarity of $d=3,4,6$ : for those values, copies of the triangle $\mathbf{T}$ create a regular tessellation of the complex plane.
[1] G. Gori and A. Trombettoni, J. Stat. Mech: Theory Exp. 2020, 063210 (2020).

[2] H. W. Diehl, Int. J. Mod. Phys. B11, 3503 (1997), condmat/9610143.

[3] K. Binder, in Phase Transitions and Critical Phenomena, edited by C. Domb (Elsevier, 2000), vol. 8, chap. 1.

[4] J. G. Brankov, D. M. Danchev, and N. S. Tonchev, Theory of Critical Phenomena in Finite-size Systems: Scaling and Quantum Effects (World Scientific, Singapore, 2000).

[5] J. L. Cardy, in Cambridge Lecture Notes in Physics (Cambridge university press, Cambridge, 1996), vol. 5.

[6] J. L. Cardy and I. Peschel, Nucl. Phys. B 300, 377 (1988), ISSN 0550-3213.

[7] J. L. Cardy, in Encyclopedia of Mathematical Physics, edited by T. S. T. J.-P. Françoise, G. L. Naber (Elsevier, 2006), pp. 333-340.

[8] O. Vasilyev, A. Gambassi, A. Maciołek, and S. Dietrich, Phys. Rev. E 79, 041142 (2009).

[9] A. Bray and M. Moore, J. Phys. A: Math. Gen. 10, 1927 (1977).

[10] H. W. Diehl and S. Dietrich, Phys. Lett. A 80, 408 (1980).

[11] H. W. Diehl and S. Dietrich, Z. Phys., B Condens. matter 42, 65 (1981).

[12] T. C. Lubensky and M. H. Rubin, Phys. Rev. B 11, 4533 (1975).

[13] D. Grüneberg and H. W. Diehl, Phys. Rev. B 77, 115409 (2008).

[14] H. W. Diehl and S. B. Rutkevich, Theor. Math. Phys. 190, 279 (2017).

[15] A. Gambassi and S. Dietrich, Europhys. Lett. 74, 754 (2006).

[16] A. Gambassi and S. Dietrich, J. Stat. Phys. 123, 929 (2006).

[17] O. Vasilyev, A. Gambassi, A. Maciołek, and S. Dietrich, Europhys. Lett. 80, 60009 (2007).

[18] M. Krech, Phys. Rev. E 56, 1642 (1997).

[19] V. M. Vassilev, D. M. Dantchev, and P. A. Djondjorov, MATEC Web Conf. 145, 01009 (2018).

[20] J. Zinn-Justin, Quantum Field Theory and Critical Phenomena (Oxford University Press, Oxford, 2002), 4th ed., ISBN 9780198509233.

[21] G. Mussardo, Statistical Field Theory: an Introduction to Exactly Solved Models in Statistical Physics (Oxford University Press, Oxford, 2020), 2nd ed., ISBN 9780198788102 .

[22] M. L. Bellac, Quantum and Statistical Field Theory (Clarendon, Oxford, 1991), 1st ed., ISBN 0198539290.

[23] D. J. Amit and V. Martin-Mayor, Field Theory, the Renormalization Group, and Critical Phenomena (World Scientific, Singapore, 2005), 3rd ed., ISBN 9812561099.

[24] H. Yamabe, Osaka J. Math. 12, 21 (1960).

[25] J. M. Lee and T. H. Parker, Bull. Am. Math. Soc. 17, 37 (1987).
[26] S.-Y. A. Chang and M. del Mar González, Adv. Math. 226, 1410 (2011), ISSN 0001-8708.

[27] K. Akutagawa, Differ. Geom. Appl. 4, 239 (1994), ISSN 0926-2245.

[28] A. L. Kholodenko and E. E. Ballard, Physica A 380, 115 (2007), ISSN 0378-4371.

[29] M. del Mar González and J. Qing, Anal. PDE 6, 1535 (2013).

[30] DLMF, NIST Digital Library of Mathematical Functions, http://dlmf.nist.gov/, Release 1.1.0 of 2020-12-15, f. W. J. Olver, A. B. Olde Daalhuis, D. W. Lozier, B. I. Schneider, R. F. Boisvert, C. W. Clark, B. R. Miller, B. V. Saunders, H. S. Cohl, and M. A. McClain, eds., URL http://dlmf .nist.gov/

[31] M. Panero and A. Smecca, J. High Energy Phys. 2021 (2021).

[32] Z. Borjan and P. J. Upton, Phys. Rev. Lett. 81, 4911 (1998).

[33] M. E. Fisher and P. J. Upton, Phys. Rev. Lett. 65, 3405 (1990).

[34] D. Amit, D. Wallace, and R. Zia, Phys. Rev. B 15, 4657 (1977).

[35] O. F. de Alcantara Bonfirm, J. E. Kirkham, and A. J. McKane, J. Phys. A: Math. Gen. 14, 2391 (1981).

[36] A. M. Polyakov, JETP Lett. 12, 381 (1970).

[37] A. M. Polyakov, Zh. Eksp. Teor. Fiz. 66, 23 (1974).

[38] A. Polyanin and V. Zaitsev, Handbook of Exact Solutions for Ordinary Differential Equations (CRC Press, Boca Raton, 1995).

[39] E. Carlon and F. Iglói, Phys. Rev. B 57, 7877 (1998).

[40] S. Rychkov, EPFL Lectures on Conformal Field Theory in $D \geq 3$ Dimensions (Springer, 2017).

[41] G. Gori and A. Trombettoni, J. Stat. Mech: Theory Exp. 2015, P07014 (2015).

[42] C. Cosme, J. V. P. Lopes, and J. Penedones, J. High Energy Phys. 2015, 1 (2015).

[43] E. Luijten, Ph.D. thesis (1997).

[44] E. Bittner, W. Janke, and H. Markum, Phys. Rev. D 66, 024008 (2002).

[45] P. H. Lundow and K. Markström, Phys. Rev. E 80, 031104 (2009).

[46] C. R. Graham and M. Zworski, Invent. Math. 152, 89 (2003).

[47] R. Zandi, A. Shackell, J. Rudnick, M. Kardar, and L. P. Chayes, Phys. Rev. E 76, 030601 (2007).

[48] Notice that in 43 the (bulk) $4 D$ Ising model at criticality is studied for linear sizes $L^{\prime}$ from 2 up to 48 (to match with our notations, the hypercube in [43] is $L^{\prime} \times L^{\prime} \times L^{\prime} \times$ $L^{\prime}$, while our slab is $\left.4 L \times 4 L \times 4 L \times L\right)$. 